\title{
Delay analysis of converged Medium Transparent fixed service Optical-Wireless networks
}

\author{
G. Kalfas ${ }^{1,3}$, J. Vardakas ${ }^{2}$, N. Pleros ${ }^{1}$, L. Alonso ${ }^{3}$, C. Verikoukis ${ }^{4}$ \\ ${ }^{1}$ Dept. of Informatics, Aristotle University of Thessaloniki, Greece \{gkalfas@csd.auth.gr, npleros@csd.auth.gr \}, \\ ${ }^{2}$ Iquadrat Informatica, Barcelona, Spain \{jvardakas@iquadrat.com $\}$,

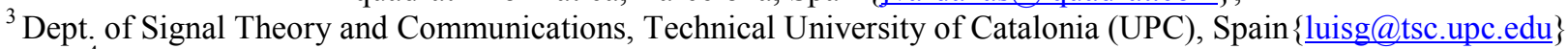 \\ ${ }^{4}$ Telecommunications Technological Centre of Catalonia (CTTC), Castelldefels, Spain \{cveri@cttc.es\}
}

\begin{abstract}
We demonstrate for the first time an analytical model for computing the end to end packet delay of an Optical/Wireless 60GHz Radio-over-Fiber (RoF) network operating under the Medium-Transparent MAC (MT-MAC) protocol. The model takes into account contention both at the optical and the wireless layer, effectively incorporating the MTMAC mechanism for seamless and dynamic capacity allocation over both optical and wireless transmission media. Based on this model, we provide an extensive delay performance analysis of the Medium Transparent MAC protocol for various optical capacity availability scenarios, varying load conditions and optical network fiber lengths. The theoretical results are found to be in good agreement with respective simulation-based findings, confirming that the employment of Medium Transparent MAC protocols can allow for efficient incorporation of the MT-MAC scheme into the upcoming era of $5 \mathrm{G}$ mm-wave small-cell networks.
\end{abstract}

Keywords-5G; Radio-over-Fiber; small cells; MediumTransparent MAC; Delay analysis; non-saturation conditions; Integrated wired/wireless access;

\section{INTRODUCTION}

Today's industrial and market leaders have proclaimed their desire to materialize the "everything in the cloud" paradigm which will offer consumers an unhindered desktop-like experience while on the move. This trend comes on top of the already exponential mobile data demand driven by the unequivocal prevalence of smart mobile devices, resulting in mobile carriers facing tremendous challenges to overcome a global bandwidth shortage[1],[2]. To this end, 5G technology is being currently developed to go far beyond that of $4 \mathrm{G}$ and adhere to a plethora of advanced requirements such as peak data rates up to $10 \mathrm{Gbps}$, cell edge data rate of $1 \mathrm{Gbps}$, mobility up to $500 \mathrm{~km} / \mathrm{h}$, a million simultaneous connections per $\mathrm{km}^{2}$, and perhaps most importantly a latency of $1 \mathrm{~ms}[3],[4]$. Recent studies have testified the excellent capacity of the mm-wave bands towards bridging the $4 \mathrm{G} / 5 \mathrm{G}$ technological gap by augmenting the currently congested sub-2.6 GHz spectrum bands employed in the telecom industry[5]-[7]. The combination of broader channel allocations that can stretch far beyond the present $20 \mathrm{MHz}$ channels used by $4 \mathrm{G}$ customers, together with matured cost-effective mm-wave CMOS technology and small high-gain steerable antennas at the mobile and base station strengthens the argument for adopting mm-wave solutions in the $5 \mathrm{G}$ era[8],[9].

The Medium Transparent MAC (MT-MAC) protocols have been proposed as efficient schemes to seamlessly converge the mm-wave small-cell wireless architecture with the optical backhaul infrastructure towards minimizing communication latency, while optimizing resource utilization[10],[11]. The MT-MAC protocols operate on the basis of a converged optical/wireless Radio-over-Fiber (RoF) system, such as the one depicted in Fig. 1, providing dynamic optical and wireless resource allocation directly between a central intelligent entity (the Central Office) and the mm-wave wireless terminals through the Remote Antenna Units (RAUs) that act solely as RF/optical signal converters. The MT-MAC protocols operate under two schemes: i)the MT-MAC that employs a fixedservice regime where each RAU is granted a static size transmission window, and, ii) the Client-Weighted MT-MAC (CW-MT-MAC) that utilizes a modified version of the gated service paradigm where each RAU is granted transmission window based on the number of terminals residing within its range. The authors have presented an analytical model for the MT-MAC[11] and CW-MT-MAC[12] protocols that enables the computation of throughput under saturation conditions, successfully confirming respective simulation results for different numbers of optical wavelength availability ratios. However, despite the framework that enables the analysis in terms of throughput under saturation conditions, there is still no theoretical framework for the non-saturated conditions, which would allow a deeper insight into the credentials of the MTMAC protocols to support the highly demanding latency requirements in $5 \mathrm{G}$ environments.

In this article, we demonstrate for the first time to our knowledge an analytical model and a detailed delay performance analysis for the MT-MAC protocol described in [10], considering various network load conditions, optical wavelength availability ratios and fiber length intervals between the Central Office (CO) and the RAUs. The proposed model relies in part on the work carried out in [13] that presents a delay analysis for the Interleaved Polling with Adaptive Cycle Time (IPACT) for Ethernet Passive Optical Networks (EPON). In order to apply to the hybrid RoF configurations, the latter model has been properly modified to 


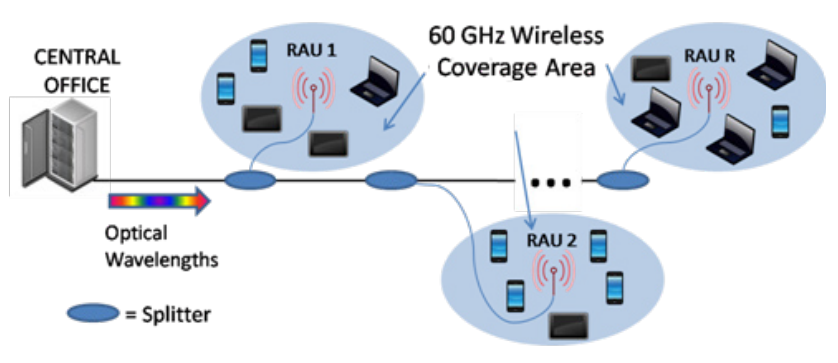

Fig. 1. Representation of an MT-MAC Radio-over-Fiber bus architecture.

account for the converged optical/wireless cycle time calculation that includes waiting times, wavelength assignments and contention in both the optical and wireless media. In this way, the proposed analysis becomes the first non saturation model to address dual resources allocation, since other state-of-the-art RoF models consider contention solely in the wireless medium with only fiber delay aspects being accounted as part of packet delay estimation[14]-[16]. The proposed analytical formula and the respective results for different loads, optical resource availability factors and fiber lengths are found to be in close agreement with simulationbased outcomes, confirming the validity of the MT-MACbased delay model. This first successful MT-MAC delay modeling approach indicates that the $\mathrm{Gb} / \mathrm{s}$-scale data-rate optical-wireless convergence yields even sub-msec delay values in non-saturation network conditions, suggesting new and efficient capacity and resource utilization concepts suitable for future $5 \mathrm{G}$ networks.

The paper is outlined as follows: Section II describes the MT-MAC protocol operational functions. Section III presents the analytical model for deriving the packet delay of the bimodal MT-MAC network. Section IV presents the performance evaluation through an extensive series of analytical and simulation results. Finally, Section V concludes the paper.

\section{Medium-Transparent MAC Protocols Review}

This section concentrates mainly on summarizing the main operational characteristics of the MT-MAC protocol that affect delay calculation. A complete description of the MTMAC protocol mechanism and its underlying $60 \mathrm{GHz} \mathrm{RoF}$ network architecture is provided in [10].

All traffic exchange takes place over both optical and wireless media with the fiber-based network delivering data from a single $\mathrm{CO}$ to multiple RAUs, while the $60 \mathrm{GHz}$ wireless interfaces contained in each RAU provide the link to the wireless terminals as shown in Fig. 1. All downlink and uplink data channels are generated at the $\mathrm{CO}$ as wavelength pairs: one wavelength is used for downlink(CO to RAU) and the other for uplink (RAU to $\mathrm{CO}$ ). A distinct wavelength pair is used as the common control channel in order to tune the RAU elements to the assigned wavelength pair that will be used for the next data transmission window. Capacity requests are directly negotiated between the terminals and the $\mathrm{CO}$ without any intelligent operation on behalf of the RAU units, thus establishing an extended reach network between all end-users served by the same CO, even for no Line-Of-Sight conditions. Access control is performed within two contention periods

This work was supported by the Marie-Curie FP7 project COMANDER (612257), Research Project AGAUR (Grant 2014-SGR-1551) and Research Project CellFive (Grant TEC2014-60130-P).

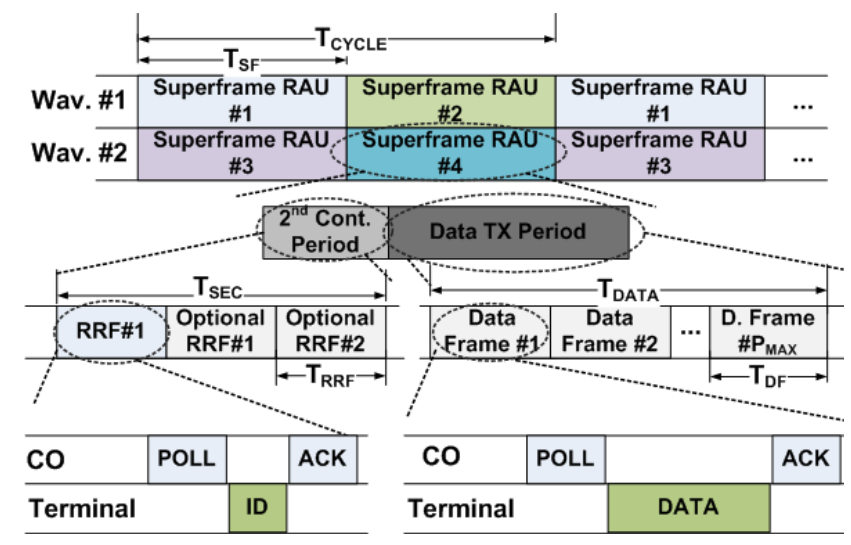

Fig. 2. Example of network with 2 wavelengths and 4 RAUs.

running in parallel: the First Contention Process (FCP) is carried out in the optical domain and informs the $\mathrm{CO}$ which RAUs contain terminals with pending traffic in their range, whereas the Second Contention Process (SCP) decides the distribution of the wireless bandwidth between end-users located in the same RAU cell.

After finalization of the FCP, the $\mathrm{CO}$ assigns a data transmission wavelength pair to each RAU. In high load conditions, where the number of RAUs containing active clients exceeds the number of available data wavelength pairs, the $\mathrm{CO}$ assigns the wavelengths in a Round-Robin fashion fairly distributing the available bandwidth amongst all RAUs. Data traffic exchange is divided logically into Superframes (SFs). Each SF, contains either Resource Requesting Frames (RRFs) that carry out the SCP process or Data Frames (DFs) that compose the DATA TX period, as shown in Fig. 2. The number of RRFs is variable and depends on the outcomes of the SCP, whereas the number of DFs is static and always equal to $P_{M A X}$ as per the MT-MAC operational rules under the fixed service regime. Each RRF is responsible for identifying the active nodes residing within the RAU cell so as to increase bandwidth utilization by allowing only active nodes' participation in the current SF. Once the contending end-users have been resolved after one or more RRFs, DFs are sequentially transmitted carrying out the actual data exchange on the basis of a polling-based scheme. RRFs are further divided into $s$ slots, with each slot being comprised of POLL, ID and ACK packets, whereas DFs contain DATA packets instead of ID packets. At the beginning of each RRF, all the active clients randomly choose an integer value $y$ in the interval $[0, s)$ where $y$ corresponds to the number of POLL packets that have to be received by the terminal before responding with an ID packet. During an RRF slot, the CO transmits a general POLL packet with no receiving node specified in its body. Upon correct ID packet reception, the $\mathrm{CO}$ responds with an ACK packet, notifying the corresponding node that it has been correctly identified. This node will not participate in a subsequent RRF (if any) within the current SF. Should, however, two nodes choose the samey value, both will then transmit an ID packet during the same slot. The collision will render both ID packets unreadable and the $\mathrm{CO}$ will not respond with an ACK, forcing the nodes to participate in the next RRF after choosing a new $y$ value. The CO continues transmitting RRFs until zero collisions occur 


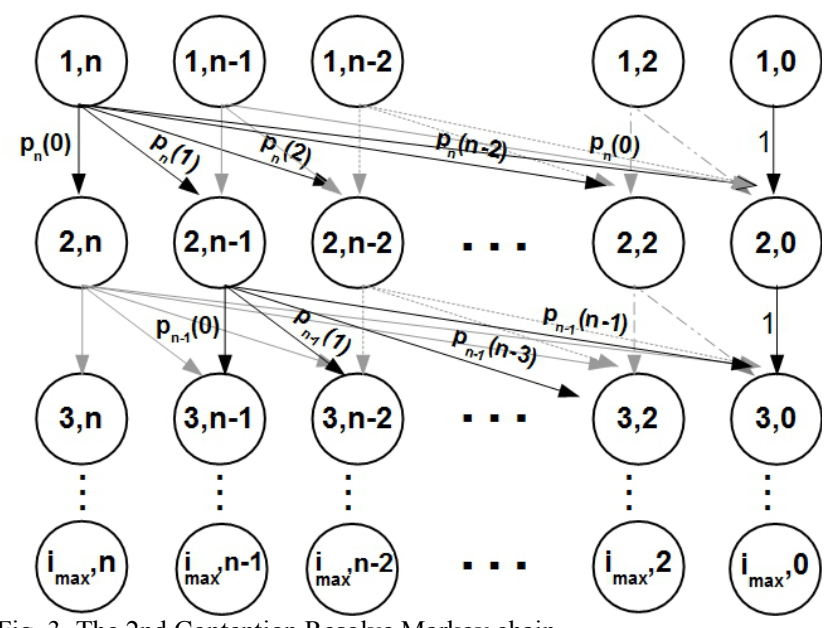

Fig. 3. The 2nd Contention Resolve Markov chain.

and therefore all nodes are successfully identified, ending in this way the SCP. Having full knowledge of the nodes that are active within a RAU, the CO initiates the DATA TX period where the main exchange of data occurs in the form of serial broadcasting of $P_{M A X}$ DF transmissions until the end of the SF is reached. In parallel to the data exchange, the $\mathrm{CO}$ periodically reruns the FCP, so as to update the list of RAUs requesting traffic. If no known active clients exist within a newly wavelength allocated RAU, the SCP is repeated.

\section{DELAY ANALYSIS}

We consider an MT-MAC network consisting of the $\mathrm{CO}, w$ wavelength pairs and $R$ RAUs connected to the $\mathrm{CO}$ through an optical fiber of length $L \mathrm{~km}$. The network is utilizing an optical bus topology and the inter-RAU fiber interval is $l \mathrm{~km}$, i.e. the first RAU is $L \mathrm{~km}$ way from the $\mathrm{CO}$, the second RAU is $L+l \mathrm{~km}$ away from the CO, etc. Data packets are assumed to follow a Poisson arrival process with bit rate of $\lambda \mathrm{Mbits} / \mathrm{s}$ and a fixed size of $B$ bits. Traffic is assumed to be symmetric, meaning that all clients introduce the same load into the network. The fiber transmission links provide $1 \mathrm{Gbit} / \mathrm{s}$ of bandwidth to each RAU which is shared amongst the wireless terminals serviced by each RAU. All RAUs are considered to serve the same number of clients $n$ and therefore the symmetric traffic property applies in the RAU domain as well. We declare $T_{\text {cycle }}$ to be the cycle time defined as the time between the start of two successive data transmission windows for a fixed RAU.

Since the MT-MAC operates under fixed service we can derive an accurate estimation of the cycle duration. To do that we need to specify the duration of the SF $T_{S F}$ and the waiting period $T_{\text {WAIT }} . T_{\text {WAIT }}$ refers to the time that a RAU has no assigned wavelengths either due to lack of pending traffic or due to wavelength time sharing amongst the network's RAUs. $T_{S F}$ is equal to the sum of the duration of the SCP $T_{S E C}$ and the data transmission period $T_{D A T A}$. The first is comprised of one mandatory RRF followed by optional RRFs in case the first does not suffice in resolving the clients that request channel access. According to the MT-MAC rules the duration of the RRF frame $T_{R R F}$ is defined as:

$$
T_{R R F}=3 \delta_{F I B E R}+s\left(3 \delta_{A I R}+T_{P O L L}+T_{I D}+T_{A C K}\right)
$$

where $\delta_{A I R}$ is the propagation delay in the wireless medium, $s$ is the size of the slots pool that the clients choose a random number from and $T_{P O L L}, T_{I D}$ and $T_{A C K}$ are the transmission delays of the POLL, ID and ACK packets respectively. On the other hand $T_{D A T A}$ is defined as the time it takes to transmit several sequential DFs plus the corresponding POLL and ACK frames. In the fixed service regime the number of sequential DFs that is granted per RAU is immutable and always equal to $P_{M A X}$. DF's duration $T_{D F}$ is defined as:

$$
T_{D F}=\frac{2 \delta_{F I B E R}+3 \delta_{A I R}+P_{M A X}\left(T_{P O L L}+T_{D A T A}+T_{A C K}\right)}{P_{M A X}}
$$

where $T_{D A T A}$ is the transmission delay of the DATA packet. Based on the above we define $T_{S F}$ as:

$$
T_{S F}=T_{R R F}+P_{M A X} T_{D F}
$$

Notice that at this point we consider only one RRF per SF. As described before, due to the MT-MAC rules this is not the only possibility since it could take more than one RRFs for the SCP to resolve. Later on, a corrective factor will be added to account for that event, but at this point only the mandatory $\mathrm{RRF}$ is taken into account. Having defined $T_{S F}, T_{\text {cycle }}$ is calculated as:

$$
T_{\text {cycle }}=\frac{R}{w} T_{S F}
$$

Since the cycle time is constant, the system can be considered at discrete moments that are apart $T_{\text {cycle }}$ seconds as depicted in the example provided in Fig. 2.

We define $Q(m)$ to be the queue size of a RAU at time $t=m * T_{\text {cycle }} \cdot Q(m)$ is a discrete homogeneous Markov chain, which means that $Q(m)$ depends on the "past" states $\left(Q_{0}, Q_{1}, \ldots, Q_{m-1}\right)$ only through the present and is independent of $m$. Therefore we are able to define transition probabilities and a transition matrix as it has been similarly done in [13]:

$$
\begin{aligned}
& p_{i, j}=\operatorname{Pr}[Q(m+1)=j \mid Q(m)=i] \\
& P=\left(\begin{array}{ccccc}
p_{0,0} & p_{0,1} & p_{0,2} & \ldots & p_{0, M} \\
p_{1,0} & p_{1,1} & p_{1,2} & \ldots & p_{1, M} \\
p_{2,0} & p_{2,1} & p_{2,2} & \ldots & p_{2, M} \\
\vdots & \vdots & \vdots & \ddots & \vdots \\
0 & 0 & 0 & \ldots & p_{M-1, M} \\
0 & 0 & 0 & \ldots & p_{M, M}
\end{array}\right)
\end{aligned}
$$

The transition probabilities are given below:

$$
p_{i, 0}=\sum_{k=0}^{P_{M A X}-i} e^{\left(-\frac{\lambda}{B} T_{c y c l e}\right)} \frac{\left(\frac{\lambda}{B} T_{c y c l e}\right)^{k}}{k !}
$$

for $i \leq P_{M A X}$,

$$
p_{i, j}=e^{\left(-\frac{\lambda}{B} T_{c y c l e}\right)} \frac{\left(\frac{\lambda}{B} T_{c y c l e}\right)^{\mathrm{P}_{\mathrm{MAX}}+j-i}}{P_{\mathrm{MAX}}+j-i}
$$

for $i \geq 0, j>0$ and $j-i \geq-P_{\mathrm{MAX}}$,

$$
p_{i, j}=0
$$


for $i, j \geq 0$ and $j-i<-P_{\mathrm{MAX}}$.

Equation (7a) states that it is only possible for a queue to send all its packets in one transmission window if the number of Poisson generated packets is equal or smaller than the maximum transmission window. This probability is given by the sum of probabilities of having no more packet arrivals than the maximum transmission window. The general probability of having a transition of $i$ packets in the queue at an instant $m$ to $j$ packet at instant $m+1$ in a cycle period is equal to the probability of generating exactly $P_{\mathrm{MAX}}+j-i$ packets and is given in(7b). Equation(7c) is a special case of (7b) and it states that if $j$ is smaller than $i-P_{M A X}$ then $p_{i, j}$ is zero since that transition would require a transmission window greater than $P_{M A X}$. In order to be able to derive results we must set the upper limit $M$ of the buffer size. This limits the dimension of the matrix $P$,causing the sum of the elements of all the lines to be less than one, which contravenes the mandatory property of a Markovian matrix. This problem is alleviated by defining the last element of each row of matrix $P$ as:

$$
p_{i, M}=1-\sum_{j=0}^{M-1} p_{i, j}
$$

In order to find the stationary probabilities of queue sizes, a linear system of equations must be solved.

$$
\begin{gathered}
\pi P=\pi \\
\sum_{i=0}^{M} \pi_{i}=1
\end{gathered}
$$

where $\pi$ is the vector defining the probabilities of the queue sizes ranging from 0 to $M$ in the steady state. The average queue size $\widehat{Q}$ at the end of each transmission cycle is:

$$
\hat{Q}=\sum_{i=0}^{M} \pi_{i} i
$$

In order to derive the average queue size $Q$ in the continuous time domain we must add the terms that account for the packets that have been produced during the SCP that precedes each SF and the packets that are born in between the discrete moments, i.e. $T_{\text {cycle }}$ intervals. For calculating the average number of RRFs that will take place at the beginning of each SF we deploy a two-dimensional (2-D) Markov chain model, depicted in Fig. 3, which demonstrates the SCP again from the perspective of a single RAU. The model follows the $S_{i, k}$ naming convention, where $i$ stands for the $i$-th RRF frame of the current SFand $k$ is the number of clients that are yet to be resolved by means of the SCP. For each SF there is an upper limit $i_{M A X}$ denoting the maximum number of RRFs that can take place within a single SF. Beyond that limit, the data transmission period commences with the clients that have been resolved, whereas any unresolved terminals will retry during the next SF.
The $2^{\text {nd }}$ Contention Markov chain can be logically divided into distinct rows and columns. The first row corresponds to the initial state of the SCP where all wireless clients are unresolved, whereas each individual row after that corresponds to a single RRF in the SF, i.e. the $2^{\text {nd }}$ row corresponds to the $1^{\text {st }}$ RRF, the $3^{\text {rd }}$ row to the $2^{\text {nd }} R R F$ and so forth. Each column represents the number of clients that are yet to be resolved in the SCP. As such, the far-left column signifies the maximum number of unresolved clients $n$ and the far-right represents the situation where all users have been resolved, i.e. the number of unresolved users has reached zero. It should be noted that $S_{i, 1}$ type of states are not present in the Markov state diagram, given that node collision can only occur when at least two users are available for picking the same slot number in the random selection process.

The initial steady state probabilities are set based on the stationary distribution of queue sizes $\pi$ :

$$
\left\{\begin{array}{c}
S_{0,0}=\pi_{0} \\
S_{i, 0}=\pi_{i, \text { for }} 0<i<n \\
S_{n, 0}=\sum_{i=n}^{M} \pi_{i \text { for }} i \geq n
\end{array}\right.
$$

The transition probability from state $S_{i, k}$ to state $S_{i+1, k-x}$ equals to the probability of having $x$ out of a current total of $k$ unresolved clients making a unique number choice and consequently getting resolved. This probability is provided by the equation:

$$
p_{k}(x)=\frac{(-1)^{x} s ! k !}{x !} \sum_{a=x}^{k} \frac{(-1)^{a}(s-a)^{k-a}}{(a-x) !(s-a) !(k-a) !}
$$

for $k \in[2, n], x \in[0, k] \backslash\{k-1\}$.

A complete summary of all the non-null one-step transition probabilities is provided below:

$$
\left\{\begin{aligned}
p\{i+1, k-x \mid i, k\} & =p_{k}(x) \\
p\{i+1,0 \mid i, 0\} & =1
\end{aligned}\right.
$$

for $\quad i \in\left[1, i_{\max }\right), k \in[0, n] \backslash\{1\}, x \in[0, k] \backslash\{k-1\}$. The second part of Eq.(14) denotes that, once reaching a state where all nodes have been resolved, the SCP is over and the CO initiates the transmission of sequential Data Frames until the end of the SF duration.

The steady state probabilities of type $S_{0, k}$ (with k>0) form the Cumulative Distribution Function (CDF) of the numbers of RRFs that are necessary for completing the SCP. Since the number of RRFs is an integer, we obtain the Probability Mass Function (PMF) from the CDF by means of subtraction. In turn, the PMF is used to derive the average number of RRFs $N_{R R F}$ necessary to complete the SCP. $N_{R R F}$ is used to calculate the average number of packets that have been produced during the SCP: 

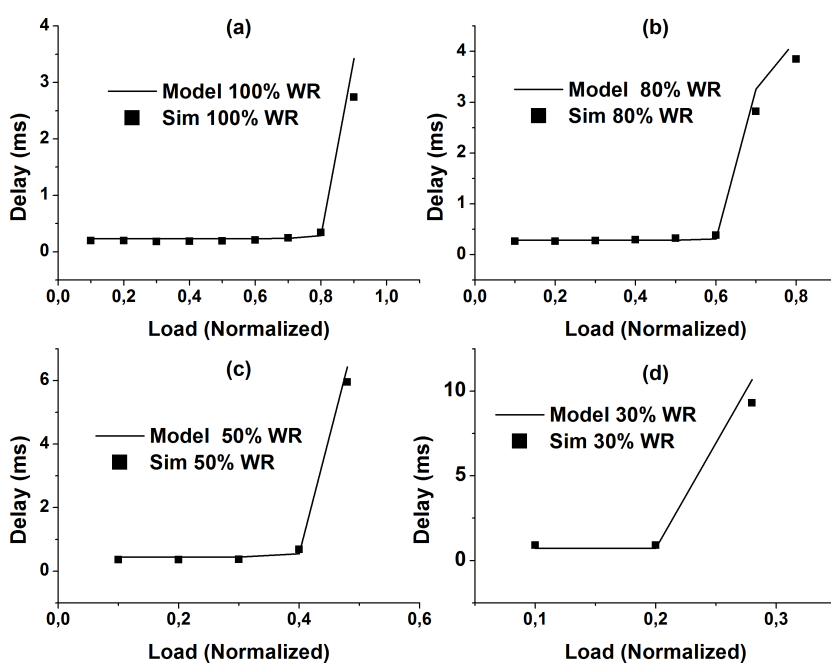

Fig.4. Delay vs. Normalized load for 10 RAUS and (a) 10 wav. (100\% WR) (b) 8 wav.(80\% WR) (c) 5 wav. $(50 \%$ WR) and (d)3 wav.(30\% WR )

$$
\begin{gathered}
Q_{R R F}=\frac{\lambda}{B}\left(N_{R R F}-1\right) T_{R R F} \text { for } N_{R R F} \geq 1 \\
Q_{R R F}=0 \text { for } N_{R R F}=1
\end{gathered}
$$

Notice that in (15a) 1 was subtracted from $N_{R R F}$, since in the initial calculation of $T_{\text {cycle }}$ the first RRF, which is mandatory, was already included.

To finalize the transition of the average queue size from the discrete to the continuous time domain we must account for the average number of packet arrivalsthat occurin between the

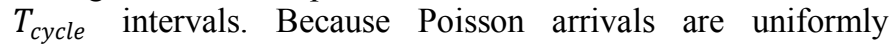
distributed in time, the average queue size in continuous time will be equal to the average queue size exactly in the middle of the discrete cycle intervals, i.e. the middle of the cycle.

$$
Q_{\text {mid }}=\frac{\lambda}{B} \frac{T_{\text {cycle }}}{2}
$$

The average queue size in continuous time $Q$ is given by the following sum:

$$
Q=\hat{Q}+Q_{R R F}+Q_{\text {mid }}
$$

By means of Little's law, which states that the average number of packets in a stable system is equal to their average arrival rate multiplied by their average waiting time in the system, we yield the average waiting time or delay $D$ :

$$
D=Q * \frac{B}{\lambda}
$$

\section{Performance EVAluation}

This section presents the performance of the proposed analytical model and evaluates its accuracy by comparing the delay values against the respective results obtained by the non saturation simulation platform employed in [10]-[12]. These results correspond to a network configuration comprising 10 RAUs in a bus topology, serviced by $w$ optical wavelength pairs each offering $1 \mathrm{Gbps}$ backhaul capacity as displayed in Fig. 1. Table I summarizes the full specification parameters of the simulation run and of the respective analytical model where applicable.

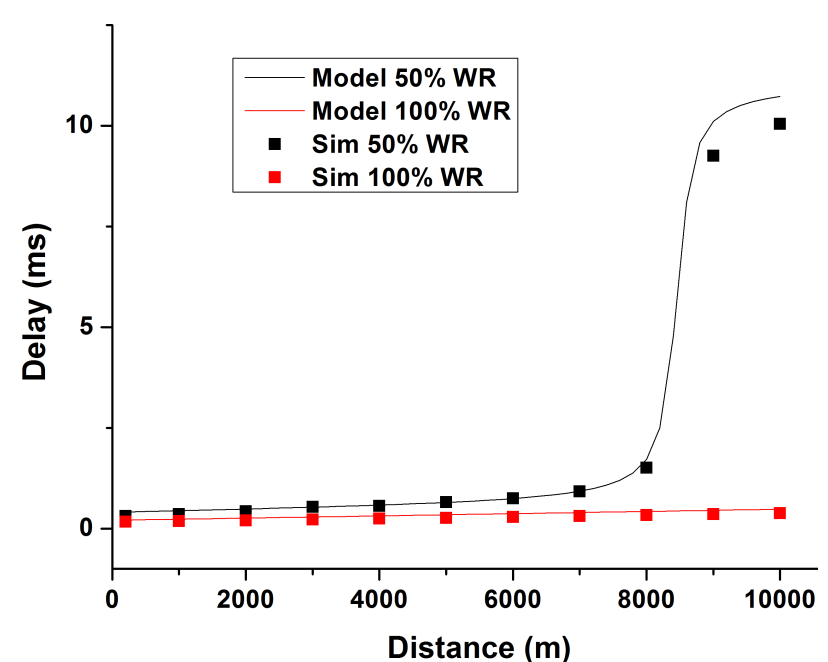

Fig. 5. Delay vs. Distance between Central Office and the 1st RAU (Fiber Length) for $50 \% \mathrm{WR}$ at $25 \%$ Norm. Load and $100 \% \mathrm{WR}$ at $100 \%$ Norm. Load.

Fig. 4 displays the packet delay versus various load conditions for four different Wavelength to RAUs Ratios (WRs) namely $30 \%, 50 \%, 80 \%$ and $100 \%$ (i.e. for 10 RAUs and 3 wavelengths WR is 30\%). Load values correspond to the aggregated Poisson arrivals of all the wireless terminals per RAU and are presented in a normalized scale compared to the wireless channel capacity, ranging from $10 \%$ (100Mbps) up to 95\% (0.95Gbps). The different WRs allow the study of the RoF system under dynamic operative conditions where the engagement of the optical resources is assigned on the fly based on the desired service level. As it is evident, the theoretical and simulation results practically coincide for all load values except in high load conditions where the network enters its saturation regime. Note that for each of the different presented WRs, the saturation pointis not constant, i.e. $30 \%$ WR the network saturates around 30\% (300Mbps) offered load per RAU, whereas for $80 \%$ WR the saturation point is around $80 \%(800 \mathrm{Mbps})$ of generated traffic. This comes as a direct effect of the backhauling capability of the presented network, since the latter depends on and is limited by the number of wavelengths available to the network. By means of Fig. 4(a) it is evident that delay values start off in the sub 0.4 millisecond range and remain very low as long as the per RAU load is under $80 \%$ ( $800 \mathrm{Mbps})$. When the offered load approaches the maximum theoretical wireless channel capacity, delay values increase rapidly as the packets remain longer in the buffer queue awaiting transmission. In this area we note also small deviations between the simulation and the analytical results. This discrepancy is explained by the fact that near the maximum theoretical wireless channel bitrate the average arrival rate approaches the system's capacity, resulting in the queue becoming unstable and therefore susceptible to small variations caused by the probabilistic Poisson traffic. The same curvature applies in the delay performance for every tested WR, as depicted in Fig. 4(b)-(d), with the only changing factor being the load value at which the network experiences saturation conditions, for reasons previously explained. The good agreement between theory and simulation confirms the validity of our proposed analytical model, while also 
confirming the low delay properties of the MT-MAC platform making it compatible for $5 \mathrm{G}$ small cell applications.

Fig. 5 presents the packet delay performance versus various fiber lengths ranging from $200 \mathrm{~m}$ up to $10 \mathrm{~km}$ for two WRs, namely $50 \%$ and $100 \%$ and for $25 \%$ and $50 \%$ normalized load generation per RAU respectively. As can be noted in the $100 \%$ WR case, delay starts at small values and increases in a linear fashion at a very low rate with respect to fiber length, but remains always at sub- $0.5 \mathrm{~ms}$ range. This small increment attests to the fact that at high optical capacity, the MT-MAC scheme achieves very efficient and fast backhauling of the wireless packets and only suffers small performance degradation as the range of the network extends up to $10 \mathrm{~km}$. Again it can be seen that the analytical and the simulation results coincide for all derived values of fiber length, since the network does not enter its saturation regime which would cause buffer depletions and instability in the queue. Regarding the $50 \%$ WR ratio, a similar performance pattern with a low rate linear increment and sub-millisecond delay values can be observed for $\mathrm{CO}$ to RAU distances ranging up to $7 \mathrm{~km}$. However in this scenario the actual delay is greater compared to the case of $100 \% \mathrm{WR}$, due to the fact that wavelength sharing causes inevitable delays as the RAUs are forced to wait approximately $50 \%$ of the system time for a wavelength assignment. When exceeding $7 \mathrm{~km}$ of fiber length however, delay values increase rapidly, as the larger cycle times cause more packet births than the amount of data frames that can be serviced by the static transmission window. This also attributes for the small discrepancy between the analytical and simulation results, since the system operates at capacity causing the utilization factor to exceed 1 so that packets are forced to wait multiple cycle times before transmission, therefore resulting in an unstable system. Despite the latter scenario however, all other analytical and simulation results confirm the MT-MAC's ability to offer an ultra-low latency small-cellular multi-Gbps front-hauling network.

TABLE I. SIMULATION SPECIFICATIONS

\begin{tabular}{|l|c|c|}
\hline \multicolumn{1}{|c|}{ Parameter } & Symbol & Value \\
\hline Number of Wavelengths & $\mathrm{w}$ & $3-10$ \\
\hline Number of RAUs & $\mathrm{R}$ & 10 \\
\hline Fiber Length between CO and 1st RAU & $\mathrm{L}$ & $200 \mathrm{~m}-10 \mathrm{~km}$ \\
\hline Packet arrival rate at the RAU & $\lambda$ & $0.1-1 \mathrm{Gbps}$ \\
\hline Fixed Transmission Window & $\mathrm{P}_{\mathrm{MAX}}$ & 30 \\
\hline Fiber propagation delay & $\delta_{\mathrm{FIBER}}$ & $1 \mu \mathrm{s}=200 \mathrm{~m}$ \\
\hline Slots in RRF & $\mathrm{s}$ & 10 \\
\hline Number of clients per RAU & $\mathrm{n}$ & 5 \\
\hline RAU range & $\mathrm{r}$ & $10 \mathrm{~m}$ \\
\hline Air propagation delay & $\delta_{\mathrm{AIR}}$ & $0,032 \mu \mathrm{s}$ \\
\hline Number of RRFs per SF & $\mathrm{N}_{\mathrm{RRF}}$ & Variable \\
\hline Wireless Data Bitrate & $\mathrm{C}$ & $1 \mathrm{Gbps}$ \\
\hline ACK Size & $\mathrm{B}_{\mathrm{ACK}}$ & 8 bytes \\
\hline DATA Packet Size (at the MAC layer) & $\mathrm{B}$ & 1500 bytes \\
\hline POLL Size & $\mathrm{B}_{\mathrm{POLL}}$ & 64 bytes \\
\hline ID Size & $\mathrm{B}_{\mathrm{ID}}$ & 64 bytes \\
\hline
\end{tabular}

\section{CONCLUSIONS}

We have demonstrated an analytical delay model for the MTMAC protocol that has been recently introduced to provide dynamic allocation of both optical and wireless resources in
60GHz RoF networks [10]. The proposed model enables the computation of end to end packet delay under non saturation conditions, successfully confirming respective simulation results for different numbers of optical wavelength availability ratios, network loads and fiber lengths. The theoretical results are found to be in excellent agreement with respective simulation-based findings, confirming that the employment of Medium Transparent MAC protocols is compatible and allows for efficient incorporation of the MT-MAC scheme into the envisioned era of $5 \mathrm{G} \mathrm{mm-wave} \mathrm{small} \mathrm{cell} \mathrm{networks.}$

\section{REFERENCES}

[1] T. S. Rappaport, J. N. Murdock, and F. Gutierrez, "State of the art in 60 $\mathrm{GHz}$ integrated circuits \& systems for wireless communications," Proc.IEEE, vol. 99, no. 8, pp. 1390-1436, Aug. 2011

[2] Z. Pi and F. Khan, "An introduction to millimeter-wave mobile broadbandsystems," IEEE Commun. Mag., vol. 49, no. 6, pp. 101-107, Jun. 2011.

[3] Recommendation ITU R M.2083-0: IMT Vision - "Framework and overall objectives of the future development of IMT for 2020 and beyond", approved Sep. 2015.

[4] A. M. Niknejad, S. Thyagarajan, E. Alon, Y. Wang and C. Hull, "A circuit designer's guide to $5 \mathrm{G}$ mm-wave," in Proc. IEEE CICC, San Jose, CA, 2015,.

[5] T. S. Rappaport et al., "Millimeter Wave Mobile Communications for 5G Cellular: It Will Work!," IEEE Access, vol. 1, no. , pp. 335-349, 2013.

[6] A. Gupta and R. K. Jha, "A Survey of 5G Network: Architecture and Emerging Technologies," in IEEE Access, vol. 3, no. , pp. 1206-1232, 2015.

[7] J. Curtis, H. Zhou, P. Hisayasu, A. Sarkar and F. Aryanfar, "MM-wave radio, a key enabler of 5G communication," 2016 IEEE 16th Topical Meeting on Silicon Monolithic Integrated Circuits in RF Systems (SiRF), Austin, TX, USA, 2016, pp. 1-3.

[8] F. Gutierrez, S. Agarwal, K. Parrish, and T. S. Rappaport, "On-chip integrated antenna structures in CMOS for $60 \mathrm{GHz}$ WPAN systems," IEEE J. Sel. Areas Commun., vol. 27, no. 8, pp. 1367-1378,Oct. 2009.

[9] T. S. Rappaport, E. Ben-Dor, J. N. Murdock, and Y. Qiao, "38 GHz and $60 \mathrm{GHz}$ Angle-dependent Propagation for Cellular and peer-to-peer wireless communications," in Proc. IEEE ICC., Jun. 2012,pp. 45684573.

[10] G.Kalfas, N. Pleros, "An Agile and Medium-Transparent MAC Protocol for $60 \mathrm{GHz}$ Radio-Over-Fiber Local Access Networks" J. of Lightwave Technol., vol.28, no.16, pp.2315-2326, Aug.15, 2010.

[11] G. Kalfas, N. Pleros, K. Tsagkaris, L. Alonso and Ch. Verikoukis, "Saturation Throughput Performance Analysis of a Medium-Transparent MAC protocol for 60GHz Radio-over-Fiber Networks", J. Lightwave Technol., vol.29, no 24, pp. 3777 - 3785, Dec.15, 2011.

[12] G. Kalfas et al., "Client-weighted medium-transparent MAC protocol for user-centric fairness in $60 \mathrm{GHz}$ radio-over-fiber WLANs," in IEEE/OSA Journal of Optical Communications and Networking, vol. 6, no. 1, pp. 33-44, Jan. 2014

[13] B. Lannoo et al., "Analytical model for the IPACT dynamic bandwidth allocation algorithm for EPONs," J. Opt. Netw. 6, 677-688 (2007)

[14] Yuting Fan et al, "Performance analysis for IEEE 802.11 distributed coordination function in radio-over-fiber-based distributed antenna systems," Opt. Express 21, 20529-20543 (2013)

[15] A. Pal and A. Nasipuri, "Performance analysis of IEEE 802.11 distributed coordination function in presence of hidden stations under non-saturated conditions with infinite buffer in radio-over-fiber wireless LANs," in Proc. 18th IEEE LANMAN, NC, Oct. 2011.

[16] M. Mjeku and N. J. Gomes, "Analysis of the request to send/clear to send exchange in WLAN over fiber networks," J. Lightwave Technol. 26(15), 2531-2539 (2008). 